\title{
TARPSY - un nouveau système tarifaire pour la psychiatrie hospitalière
}

\section{Matthias Hilpert}

Observateur de la FMH dans le groupe de pilotage TARPSY

1 Andreas S, Dirmaier J, Koch U, Schulz H. DRG-Systeme in der Versorgung von Patienten mit psychischen Störungen: Zur Konzeption eines Klassifikationssystems für Fallgruppen. Fortschr Neurol Psychiat. 2003;71: 234-42.

2 Warnke I, Rössler W. Length oft he stay by ICD-based diagnostic groups as a basis for the remuneration of psychiatric impatient care in Switzerland? Swiss Med Wkly. 2008;(35/36):520-7.

3 Décision du Conseil d'administration de SwissDRG SA du 9 novembre 2010 (www.swissdrg.org/ assets/pdf/DA_SwissDRG-VRBeschluss_PsychReha_20101111_f.pdf)

Correspondance: Dr Matthias Hilpert Services psychiatriques Argovie AG Case postale 432 CH-5201 Brugg

matthias.hilpert[at]pdag.ch
Le 21 décembre 2007, le Parlement a accepté une révision partielle de la loi fédérale sur l'assurancemaladie (LAMal) dans le domaine du financement des hôpitaux. Parmi les principaux changements figure le passage du financement de l'hôpital au financement des prestations fournies par l'hôpital. Alors que les traitements psychiatriques stationnaires font aujourd'hui encore l'objet de forfaits journaliers uniformes, les cliniques devront à l'avenir être indemnisées par une combinaison de forfaits journaliers et de forfaits par cas différenciés. A cet effet, des forfaits liés aux prestations basés sur des structures tarifaires uniformes pour l'ensemble de la Suisse seront introduits.

Tout cela existe déjà dans les hôpitaux de soins somatiques aigus depuis l'introduction des DRG. Mais ce système d'indemnisation reposant en priorité sur les diagnostics et les procédures ne convient pas à la psychiatrie [1]. La durée moyenne de séjour dans une clinique psychiatrique est en effet bien plus individuelle que dans d'autres disciplines médicales. Ainsi le traitement hospitalier d'un patient diagnostiqué schizophrène peut durer quelques jours ou prendre jusqu'à six mois. Cette variance extrême de la durée des séjours ne permet pas de calculer un forfait par cas en fonction du diagnostic [2]. A ce jour, il n'existe aucun système tarifaire basé sur les prestations adapté aux traitements psychiatriques hospitaliers, d'où la nécessité de développer un nouveau système tarifaire.

Avec leurs projets respectifs «Psysuisse» et «P-TAR», l'association H+ Les hôpitaux de Suisse et la Direction de la santé publique du canton de Zurich ont tout d'abord développé chacun de leur côté deux modèles indépendants. En 2011, elles ont fusionné leurs travaux et lancé leur projet commun TARPSY (voir également le bulletin d'information TARPSY du 3 octobre 2011). Alors que la direction stratégique est assumée par SwissDRG SA, H+ et la Direction de la santé publique du canton de Zurich se partagent la direction opérationnelle du projet TARPSY. Ainsi le projet dispose maintenant d'un mandat formel sur l'ensemble du territoire pour élaborer un système tarifaire lié aux prestations pour la psychiatrie hospitalière [3].

\section{Objectifs pour le développement} du nouveau modèle tarifaire

Les exigences envers TARPSY sont multiples. Le nouveau modèle tarifaire doit être un système de rémunération par cas qui prend en compte les prestations fournies tout en créant des incitatifs positifs. Il doit également éviter les incitatifs erronés. Il faut que la diminution de la durée d'hospitalisation pour une qualité de traitement identique soit récompensée, ce qui ne sera possible que si l'on peut mesurer la qualité. Le nouveau modèle doit par ailleurs répondre à une condition importante, à savoir que le travail requis pour relever les données ainsi que le nombre de groupes de cas ayant une incidence sur le tarif doivent rester dans une mesure raisonnable. Un objectif complémentaire est de limiter les risques de manipulation. Enfin, l'application du modèle tarifaire doit être simple et réaliste, et le travail requis pour documenter les cas réduit au strict minimum.

Le nouveau modèle tarifaire TARPSY se veut être un système évolutif. Dans ce sens, il doit être modulable, facilement vérifiable et apte à être modifié. De plus, il doit permettre de mettre en place des comparaisons entre les cliniques.

Ces objectifs sont à mettre en œuvre dans le nouveau modèle tarifaire de telle sorte que les prestataires de soins, les assureurs et autres répondants des coûts l'acceptent.

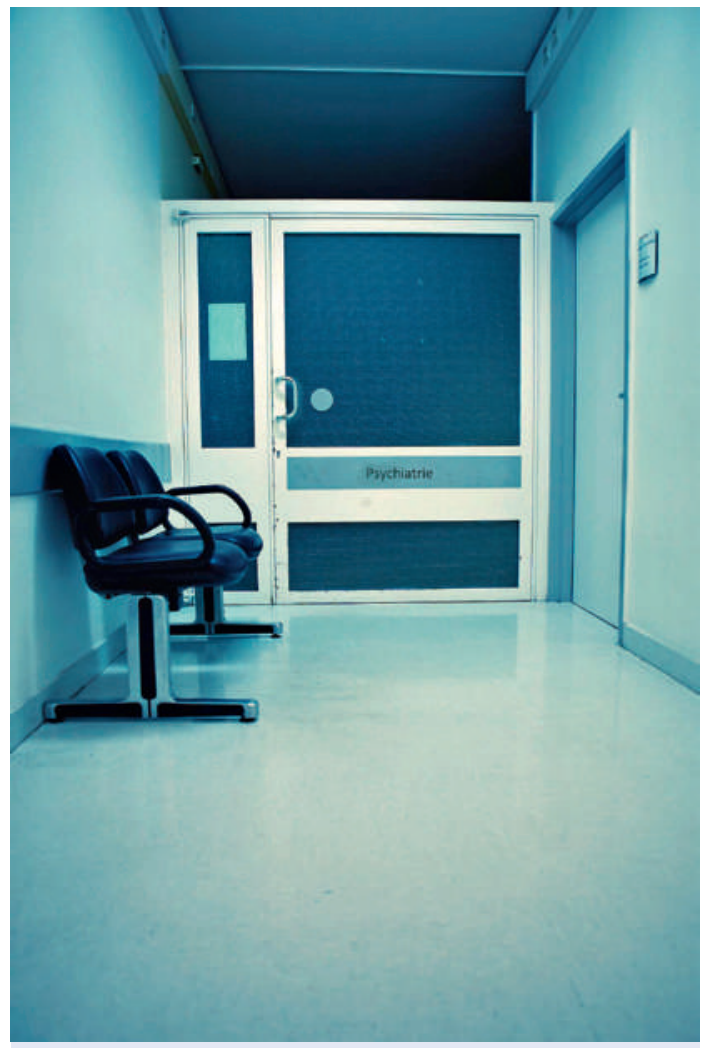

L'échéancier prévoit une mise en application de TARPSY pour la première fois en 2015. 


\section{Le diagnostic et la gravité du cas définissent les différents groupes de cas (Psychiatric Cost Groups, PCG)}

L'évaluation des données de coûts déjà disponibles a montré qu'en psychiatrie, il n'existe pas de différences de coûts marquantes entre tous les groupes de diagnostic, de traitement ou d'âge. Cependant, il semble nécessaire et important de différencier certains groupes de diagnostic principal et de faire la différence entre traitement ordinaire et traitement intensif. Certains groupes de diagnostic ont des coûts par cas plus importants, p. ex. les maladies addictives, en raison notamment de différences nettes dans la durée de séjour.

La variance expliquée sera plus faible dans le domaine de la psychiatrie que dans celui des soins somatiques aigus. Les variances en termes de coût s'expliquent par l'influence de la gravité des cas sur les coûts journaliers et les coûts par cas. Ces connaissances seront intégrées au développement du tarif dans le but de poser la base d'un regroupement simplifié des patients avec le moins possible de PCG (Psychiatric Cost Groups). Les PCG seront déterminés en combinant les principaux groupes de diagnostic conformément à la CIM-10 GM et la gravité du cas. Par ailleurs, des catégories spécifiques seront créées pour les groupes d'âge enfants (jusqu'à 14 ans) et adolescents (14 à 18 ans), et pour les patients de la forensique (exécution des peines et mesures).

\section{Evaluation de la qualité}

\section{dans le nouveau modèle tarifaire}

Pour le monitorage de la qualité, l'équipe de projet travaille en collaboration avec l'Association nationale pour le développement de la qualité dans les hôpitaux et les cliniques (ANQ). En 2011, l'ANQ a publié des normes nationales pour évaluer la qualité. Depuis juillet 2012, toutes les cliniques psychiatriques de Suisse effectuent des relevés relatifs à la qualité selon les normes de l'ANQ. L'application des mêmes méthodes au nouveau modèle tarifaire permettra d'utiliser les synergies afin de réduire considérablement les coûts de la saisie des données.

La gravité des cas (répartie en traitement ordinaire et traitement intensif) est déterminée à intervalles réguliers (7 jours) par l'intermédiaire d'HONOS (Health of the National Outcome Scales). Parallèlement, il est prévu d'effectuer un relevé à l'aide du BSCL (Brief Symptom Checklist), un instrument d'auto-évaluation qui doit réduire les risques de manipulation. En comparant les valeurs de ces deux échelles, la plausibilité des données sur la gravité peut être vérifiée.

priées de s'adresser aux responsables du projet: Urs Schönenberger, $\mathrm{H}+\mathrm{les}$ hôpitaux de Suisse, ou Janine Landtwing, Direction de la santé publique du canton de Zurich.

\section{Cliniques pilotes du projet TARPSY}

Afin d'obtenir les données indispensables au développement du modèle tarifaire, l'équipe de projet collabore avec plusieurs cliniques. Ces cliniques pilotes mettent à disposition des fichiers de données déterminés, qui contiennent les données de la statistique médicale de l'OFS, les données servant à évaluer la gravité du cas, la durée des prestations fournies par les divers groupes professionnels, les données relatives aux périodes de congé et les données des coûts par cas. Les représentants des cliniques pilotes peuvent être impliqués lors de l'élaboration de détails importants, comme p. ex. lors de questions concernant la définition du cas, le regroupement des cas et la réglementation lors de réadmission. De manière générale, les cliniques pilotes disposent d'une longueur d'avance en termes de connaissances*.

\section{Domaine d'application de TARPSY}

Le futur modèle tarifaire couvrira l'ensemble des traitements psychiatriques hospitaliers, tous les groupes de diagnostic et tous les groupes d'âge ainsi que tous les types de cliniques et tous les domaines spécialisés (intervention en cas de crise, forensique, psychiatrie pour enfants et adolescents, psychothérapie, réadaptation, etc.).

Pour que le nouveau modèle tarifaire puisse être appliqué par une clinique ou la division d'une clinique, celle-ci doit avoir un mandat de prestations pour la psychiatrie, conformément à la liste cantonale des hôpitaux. La séparation entre coûts et prestations doit être transparente. Cela signifie que l'on doit pouvoir contrôler de manière détaillée les prestations hospitalières fournies pour traiter chaque patient ainsi que les dépenses que l'hôpital a engagées à cet effet. Pour qu'un cas puisse être indemnisé avec TARPSY, il faut que le diagnostic principal soit psychiatrique.

Suite à la révision de la LAMal, la catégorie de traitement «semi-hospitalier» a été supprimée. Désormais, on ne distingue plus que les traitements ambulatoires et stationnaires. Or le TARMED, le tarif appliqué pour les soins ambulatoires, n'est que partiellement adapté aux hôpitaux psychiatriques de jour et de nuit, car les prestations des différents groupes professionnels ne peuvent pas être facturées correctement. Un avant-projet devra donc examiner si les traitements des hôpitaux psychiatriques de jour et de nuit pourront également être intégrés dans le modèle TARPSY.

\section{Perspectives}

Jusqu'à l'entrée en vigueur du nouveau modèle tarifaire, il reste beaucoup à faire. Les fichiers de données reçus doivent être analysés afin d'en déduire les PCG, les structures tarifaires et les règles. Il faut également établir les directives concernant le codage et développer un logiciel pour grouper les données et calculer les forfaits individuels. Il s'agit également de créer une grille pour comparer les cliniques. Et enfin, le modèle tarifaire doit être approuvé par le Conseil fédéral. L'échéancier prévoit une mise en application

de TARPSY pour la première fois en 2015. 